\title{
Iron and Neurodegeneration in Multiple Sclerosis
}

\author{
Michael Khalil, ${ }^{1,2}$ Charlotte Teunissen, ${ }^{2}$ and Christian Langkammer1 \\ ${ }^{1}$ Department of Neurology, Medical University of Graz, A-8036 Graz, Austria \\ ${ }^{2}$ NUBIN, Department of Clinical Chemistry, VU University Medical Center, 1007MB Amsterdam, The Netherlands
}

Correspondence should be addressed to Michael Khalil, michael.khalil@medunigraz.at

Received 31 October 2010; Revised 26 December 2010; Accepted 2 January 2011

Academic Editor: Jeroen J. G. Geurts

Copyright () 2011 Michael Khalil et al. This is an open access article distributed under the Creative Commons Attribution License, which permits unrestricted use, distribution, and reproduction in any medium, provided the original work is properly cited.

\begin{abstract}
Increased iron deposition might be implicated in multiple sclerosis (MS). Recent development of MRI enabled to determine brain iron levels in a quantitative manner, which has put more interest on studying the role of iron in MS. Evidence for abnormal iron homeostasis in MS comes also from analyses of iron and iron-related proteins in CSF and blood and postmortem MS brain sections. However, it is not yet clear if iron accumulation is implicated in MS pathology or merely reflects an epiphenomenon. Further interest has been generated by the idea of chronic cerebrospinal venous insufficiency that might be associated with brain iron accumulation due to a reduction in venous outflow, but its existence and etiologic role in MS are currently controversially debated. In future studies, combined approaches applying quantitative MRI together with CSF and serum analyses of iron and iron-related proteins in a clinical followup setting might help to elucidate the implication of iron accumulation in MS.
\end{abstract}

\section{Introduction}

Iron is essential for normal neuronal metabolism, including mitochondrial energy generation and myelination $[1,2]$. However, excessive levels of brain iron may exert ironinduced oxidative stress and thus lead to neurodegeneration [3]. During the process of normal aging, various regions of the brain, predominantly the basal ganglia, tend to accumulate nonhemin iron, which is primarily stored in the form of ferritin [4]. Increased iron deposition has been observed in various chronic neurological disorders, including multiple sclerosis (MS) [5].

Evidence for increased iron accumulation in MS is mainly derived from magnetic resonance imaging (MRI) and histopathologic studies; however, some information exists also from analyses of iron and iron-related proteins in cerebrospinal fluid (CSF) and blood. The following review summarizes current knowledge of increased brain iron accumulation in MS derived from (2) MRI, (3) histopathologic analyses, (4) studies on CSF and blood, and (5), finally, provides an outlook on potential therapeutic interventions.

\section{Magnetic Resonance Imaging}

In several studies, evidence for increased iron accumulation, preferentially in deep gray matter areas of the brain, was mainly derived from the signal reduction on T2-weighted MR images [5].

First reports on a regionally signal reduction on T2weighted brain MRI images in MS indicative of increased iron deposition were published by Drayer et al. [6] and Grimaud et al. [7].

Several studies then followed with a focus on the clinical implication of increased iron accumulation in MS. Increased deep gray matter T2 hypointensities were found to be correlated with disease duration $[8,9]$, physical disability [9-13], and cognitive impairment [14]. Clinical followup studies in MS revealed that baseline gray matter T2 hypointensities were associated with disability progression over time $[12,15]$. Another consistent finding is that deep gray matter T2 hypointensity, suggestive of increased iron content, is correlated with brain atrophy $[8,16]$. While this was evidenced in patients with definite MS, there is only little information available regarding the extent and clinical significance of increased iron deposition in patients with a clinically isolated syndrome. Ceccarelli et al. found only minor changes of signal reductions on T2-weighted images compared to healthy controls, and the extent did not predict conversion to clinically definite MS [17]. The approaches used in the studies mentioned above suffered from the methodological drawback of deducing iron concentrations 
from a visual grading of the reduction of signal intensity on T2-weighted images even though more recent studies have determined the extent of $\mathrm{T} 2$ hypointensity in a semiquantitative manner $[8,10,14,16]$.

In recent years, methodical development of MRI enabled to assess brain iron concentrations quantitatively. In addition, quantitative iron mapping by MRI offers a more sensitive discrimination of iron levels and, therefore, is especially advantageous in longitudinal studies and monitoring of long-term disease progression.

The techniques utilized for quantitative iron mapping are mainly based on relaxation time mapping [18-20] (Figure 1) but also other approaches such as phase mapping [21, 22], magnetic field correlation [23], or direct saturation imaging [24] are applied.

Susceptibility weighted imaging (SWI), a technique that takes advantage from the full complex MR signal by combining magnitude and phase images, has gained attention as a means to assess brain iron $[25,26]$. However, the complexity of the postprocessing involved in SWI renders comparative studies challenging and remains an objective of research [27]. Quantitative susceptibility mapping (QSM) is an approach using solely phase images and produces susceptibility maps which are independent of the orientation of the tissue to the main magnetic field $[28,29]$. Because paramagnetic iron is considered a main determinant of brain tissue susceptibility, QSM seems especially useful to assess brain iron.

2.1. Validation of MRI Methods. Several methods have been proposed for the measurement of brain iron concentration; however, the majority of them lack validation and, therefore, the specificity and sensitivity of these techniques are not reliably known.

From theoretical considerations based on susceptibility models for brain tissue, it can be concluded that iron is a main determinant of susceptibility-induced contrast in MRI [30]. Several studies have indirectly investigated the relation of MRI parameters with iron by using the age-dependency of iron accumulation in the basal ganglia as reported in $[4,31]$.

Recently, high-pass filtered SWI phase images were compared to regional iron concentrations in postmortem tissue determined by synchrotron X-ray fluorescence and revealed a correlation between phase shifts and iron [32].

Other recent work acquired quantitative MRI directly after death from seven human brains and subsequently determined brain iron concentrations by using inductively coupled plasma mass spectrometry [33]. This study showed that the relaxation rates $\mathrm{R} 2$ and $\mathrm{R} 2 *$ can be used as sensitive and linear measures for brain iron concentration.

These quantitative MRI techniques together with a better understanding of pathophysiologic concepts of increased iron levels [1-3] have put more interest on elucidating the role of iron in MS.

In recently performed studies on quantitative brain iron levels in MS, based on R2* relaxometry at 3 Tesla, increased iron levels have been found in patients with advancing MS compared to clinically isolated syndrome [20]. Using this validated quantitative technique, higher R2* levels in basal ganglia structures reflecting higher iron content were

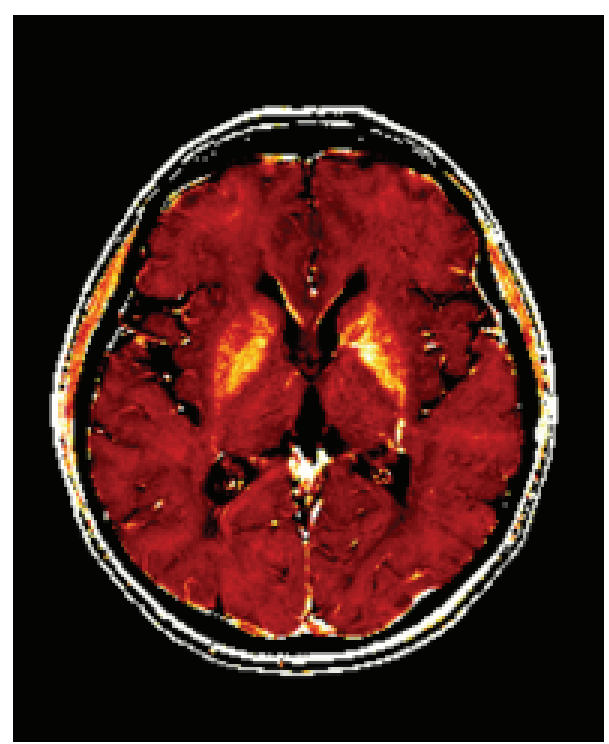

FIGURE 1: R2* map of a 50-year-old female MS patient. Higher iron concentrations in basal ganglia structures are reflected by brighter signal intensities.

correlated with gray matter atrophy and also with T2-lesion volume [20]. These findings are supported by earlier studies where MRI T2 hypointensities suggestive of increased brain iron, preferentially located in deep gray matter areas, were linked to physical disability and gray mater atrophy in MS [8-10, 12, 34]. Further support comes from a followup study showing that MRI T2 shortenings in deep gray matter areas at baseline are predictive of the evolution of brain atrophy [16].

Apart from gray matter regions with known high iron levels (putamen, globus pallidus, caudate nucleus, substantia nigra, and red nucleus) efforts were made to investigate iron levels in white matter by MRI [22, 35, 36]. Using SWI, the phase values of MS lesions were investigated and compared to adjacent white matter [36]. However, compared with chemically determined iron concentrations of postmortem studies, the iron levels within MS lesions were not substantially altered than in reference white matter structures $[4,33]$. Due to the confounding impacts of iron and myelin to MRI contrast generation, disease-induced alterations of iron levels in white matter need to be treated with caution and are an objective of ongoing research [37].

Further interest on iron deposition in MS has been generated by the idea of chronic cerebrospinal venous insufficiency (CCSVI) [38] that might be associated with the accumulation of iron in the brain due to a reduction in venous outflow [39, 40]. Following this hypothesis, CCSVI is postulated to be implicated in the etiology of MS. The underlying mechanism is believed to originate from increased iron accumulation in patients due to a reduced venous blood flow caused by constrictions of cerebral veins. This then leads to extravasation of erythrocytes with subsequent iron deposition [41], subsequently triggering inflammationdependent tissue damage [42]. However, the existence of 
CCSVI as well as its etiologic role in MS are currently controversially debated [43], and there is an increasing amount of papers published now that challenge this hypothesis [44-47]. Furthermore, histopathologic studies do not provide clear evidence for extravasation of erythrocytes into lesions caused by increased intraluminal venous pressure [48-52].

\section{Histopathology and Pathologic Significance of Increased Brain Iron}

The normal anatomic and cellular age-dependent iron distribution within the brain, as described previously [4, 53, 54], should be considered when comparing with iron deposition in pathological conditions.

Craelius et al. described positive iron staining in MS brain sections surrounding demyelinated plaques, myelinated white matter near the lesions, and within blood vessels of gray matter near the lesion [55]. Iron deposits were also described in the putamen and the thalamus [6], in macrophages and reactive microglia [56] and in normalappearing white matter tissue [57]. Mehindate et al. showed that heme oxygenase 1 , which is involved in regulating iron metabolism, was upregulated in astrocytes of MS spinal cord tissue [58].

The exact underlying mechanism by which brain iron accumulates in MS is not fully understood. Iron transport across the blood-brain barrier is dependent on iron transport proteins, predominantly by transferring receptors expressed on brain epithelial cells [59]. Other transporters may also facilitate iron transport across the blood-brain barrier, such as the divalent metal transporter (DMT) and the lactoferrin receptor [60].

It is also not yet clear if increased brain iron deposition is implicated in MS pathology or merely reflects an epiphenomenon $[3,61]$. Potential toxic iron products may arise when hydrogen peroxide is formed by superoxide dismutase, which then reacts with free or poorly liganded iron (Fenton reaction [62]). Superoxide may also react with ferric iron through the Haber-Weiss reaction, producing $\mathrm{Fe}^{2+}$, which then again affects the redox cycling $[1,2]$ (Figure 2).

The resulting highly reactive free hydroxyl radicals $\left(\mathrm{OH}^{\bullet}\right)$ interact with molecules leading to the production of other free radicals [63]. This leads to oxidative stress-induced lipid peroxidation, mitochondrial dysfunction, increase in intracellular free-calcium concentration, and finally causing cell dysfunction and death [62-64]. Because neuronal membrane lipids are rich in highly polyunsaturated fatty acid, they are susceptible to damage caused by lipid peroxidation $[62,63]$. Iron itself can initiate and amplify lipid peroxidation $[62,63]$. Several naturally produced antioxidants, such as alphatocopherol, may help to reduce oxidative stress-induced tissue damage [62].

\section{Cerebrospinal Fluid and Blood}

Only a limited number of studies have analyzed iron and iron-related protein levels in CSF and peripheral blood of MS patients. CSF ferritin levels were shown to be elevated in patients with chronic progressive active MS [65] and in patients with SPMS compared to controls [46, 57]. Another study showed that CSF ferritin levels were lower but within normal limits in patients with optic neuritis compared to patients with other neurologic diseases [66]. Similar levels of CSF ferritin were detected in RRMS patients compared to controls $[57,67]$. In a recently performed cross-sectional and longitudinal study, CSF ferritin levels did not significantly change over a time period of 3 years, which also may argue against an etiologic role for CCSVI-related parenchymal iron deposition in MS [46].

Serum soluble transferring-receptor levels were significantly increased in MS compared to controls [68, 69], while serum ferritin levels were elevated in patients with chronic active MS only [68]. Conversely, analyses of iron status in two children with recurrent episodes of tumefactive cerebral demyelination revealed decreased serum iron and ferritin and constant iron supplementation was needed to prevent an iron deficiency state in both children [70].

\section{Therapeutic Implications}

On basis of pathophysiologic concepts implicating ironinduced tissue damage in MS, potential therapeutic interventions, including iron chelators, and inhibitors of ironrelated oxidative stress and lipid peroxidation may have beneficial effects $[3,71,72]$. Several chelators are of putative therapeutic value in neurodegenerative disorders [73].

Studies on experimental autoimmune encephalomyelitis (EAE), the animal model of MS, showed that treatment with the iron chelator desferrioxamine reduced clinical and pathologic signs of EAE [74]. Deferiprone, an orally delivered iron chelator, ameliorated signs of EAE, an inhibited Tcell function [75]. However, a clinical trial testing the iron chelating drug desferrioxamine in chronic progressive MS patients failed to demonstrate any effects on disease progression [76]. A recent observation revealed that supplementing nonanaemic iron deficiency in two children with recurrent episodes of tumefactive demyelination leads to sustained remission [70].

In the future large randomized double-blinded multicenter studies are needed to elucidate the potential use of therapies targeting oxidative stress and lipid peroxidation in patients with MS. Quantitative MRI techniques and detailed monitoring of body-fluid iron and iron-related proteins levels should be included in such study protocols.

\section{Summary}

In summary, increased iron deposition has been consistently reported to occur in MS, but its role in pathogenetic processes of this disease has not yet been completely clarified. Whether increased brain iron levels are also the cause or only the consequence of tissue destruction is still a matter of debate. Future longitudinal studies combining clinical disease status, quantitative MRI techniques sensitive for iron, and additional analyses of iron in CSF/serum and iron-related proteins (as well as iron regulator proteins), might help to unravel the implication of increased iron accumulation in MS. Quantitative MRI and histopathologic 


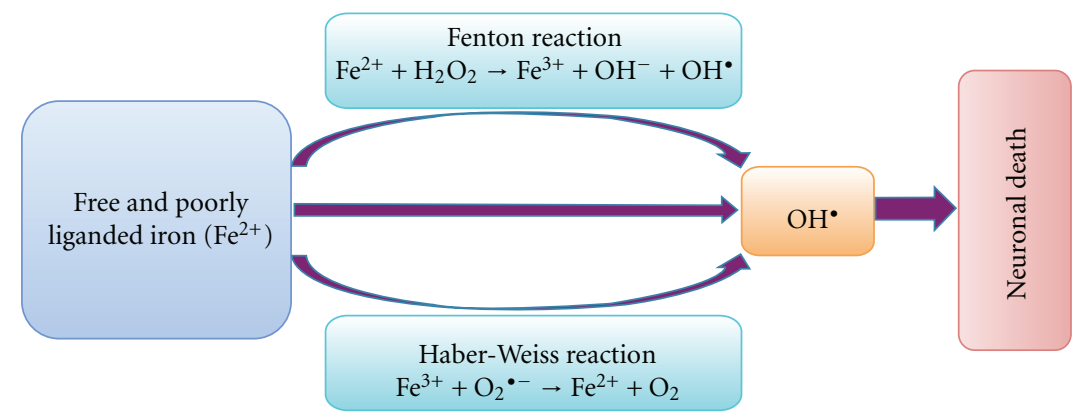

FIGURE 2: Generation of reactive and damaging hydroxyl radicals $\left(\mathrm{OH}^{\bullet}\right)$. Free Iron $\left(\mathrm{Fe}^{2+}\right)$ reacts trough the Fenton reaction with hydrogen peroxide, leading to the generation of very reactive and damaging hydroxyl radicals $\left(\mathrm{OH}^{\bullet}\right)$. Superoxide can also react with ferric iron in the Haber-Weiss reaction leading to the production of $\mathrm{Fe}^{2+}$, which then again affects redox cycling. The highly reactive hydroxyl radicals lead to oxidative stress-induced lipid peroxidation, mitochondrial dysfunction, and increase in intracellular free-calcium concentration, and finally causing neuronal death.

analyses of postmortem MS brains should complement these studies.

\section{Acknowledgment}

M. Khalil received research support from the Austrian Science Fund (FWF) [J2992-B09].

\section{References}

[1] D. B. Kell, “Towards a unifying, systems biology understanding of large-scale cellular death and destruction caused by poorly liganded iron: Parkinson's, Huntington's, Alzheimer's, prions, bactericides, chemical toxicology and others as examples," Archives of Toxicology, vol. 84, no. 11, pp. 825-889, 2010.

[2] D. B. Kell, "Iron behaving badly: inappropriate iron chelation as a major contributor to the aetiology of vascular and other progressive inflammatory and degenerative diseases," $B M C$ Medical Genomics, vol. 2, article 2, 2009.

[3] L. Zecca, M. B. H. Youdim, P. Riederer, J. R. Connor, and R. R. Crichton, "Iron, brain ageing and neurodegenerative disorders," Nature Reviews Neuroscience, vol. 5, no. 11, pp. 863-873, 2004.

[4] B. Hallgren and P. Sourander, "The effect of age on the nonhaemin iron in the human brain," Journal of Neurochemistry, vol. 3, pp. 41-51, 1958.

[5] J. Stankiewicz, S. S. Panter, M. Neema, A. Arora, C. E. Batt, and R. Bakshi, "Iron in chronic brain disorders: imaging and neurotherapeutic implications," Neurotherapeutics, vol. 4, no. 3, pp. 371-386, 2007.

[6] B. Drayer, P. Burger, and B. Hurwitz, "Reduced signal intensity on MR images of thalamus and putamen in multiple sclerosis: increased iron content?" American Journal of Roentgenology, vol. 149, no. 2, pp. 357-363, 1987.

[7] J. Grimaud, J. Millar, J. W. Thorpe, I. F. Moseley, W. I. McDonald, and D. H. Miller, "Signal intensity on MRI of basal ganglia in multiple sclerosis," Journal of Neurology Neurosurgery and Psychiatry, vol. 59, no. 3, pp. 306-308, 1995.

[8] R. Bakshi, R. H. B. Benedict, R. A. Bermel et al., "T2 hypointensity in the deep gray matter of patients with multiple sclerosis: a quantitative magnetic resonance imaging study," Archives of Neurology, vol. 59, no. 1, pp. 62-68, 2002.
[9] R. Bakshi, Z. A. Shaikh, and V. Janardhan, "MRI T2 shortening ('black T2') in multiple sclerosis: frequency, location, and clinical correlation," NeuroReport, vol. 11, no. 1, pp. 15-21, 2000.

[10] C. W. Tjoa, R. H. B. Benedict, B. Weinstock-Guttman, A. J. Fabiano, and R. Bakshi, "MRI T2 hypointensity of the dentate nucleus is related to ambulatory impairment in multiple sclerosis," Journal of the Neurological Sciences, vol. 234, no. 1-2, pp. 17-24, 2005.

[11] Y. Zhang, R. K. Zabad, X. Wei, L. M. Metz, M. D. Hill, and J. R. Mitchell, "Deep grey matter "black T2" on 3 tesla magnetic resonance imaging correlates with disability in multiple sclerosis," Multiple Sclerosis, vol. 13, no. 7, pp. 880883, 2007.

[12] Y. Zhang, L. M. Metz, V. W. Yong, and J. R. Mitchell, “3 T deep gray matter T2 hypointensity correlates with disability over time in stable relapsing-remitting multiple sclerosis: a 3-year pilot study," Journal of the Neurological Sciences, vol. 297, no. 1-2, pp. 76-81, 2010.

[13] A. Ceccarelli, M. Filippi, M. Neema et al., "T2 hypointensity in the deep gray matter of patients with benign multiple sclerosis," Multiple Sclerosis, vol. 15, no. 6, pp. 678-686, 2009.

[14] S. D. Brass, R. H. B. Benedict, B. Weinstock-Guttman, F. Munschauer, and R. Bakshi, "Cognitive impairment is associated with subcortical magnetic resonance imaging grey matter T2 hypointensity in multiple sclerosis," Multiple Sclerosis, vol. 12, no. 4, pp. 437-444, 2006.

[15] M. Neema, A. Arora, B. C. Healy et al., "Deep gray matter involvement on brain MRI scans is associated with clinical progression in multiple sclerosis," Journal of Neuroimaging, vol. 19, no. 1, pp. 3-8, 2009.

[16] R. A. Bermel, S. R. Puli, R. A. Rudick et al., "Prediction of longitudinal brain atrophy in multiple sclerosis by gray matter magnetic resonance imaging T2 hypointensity," Archives of Neurology, vol. 62, no. 9, pp. 1371-1376, 2005.

[17] A. Ceccarelli, M. A. Rocca, M. Neema et al., "Deep gray matter T2 hypointensity is present in patients with clinically isolated syndromes suggestive of multiple sclerosis," Multiple Sclerosis, vol. 16, no. 1, pp. 39-44, 2010.

[18] E. M. Haacke, N. Y. C. Cheng, M. J. House et al., "Imaging iron stores in the brain using magnetic resonance imaging," Magnetic Resonance Imaging, vol. 23, no. 1, pp. 1-25, 2005. 
[19] A. L. MacKay, I. M. Vavasour, A. Rauscher et al., "MR relaxation in multiple sclerosis," Neuroimaging Clinics of North America, vol. 19, no. 1, pp. 1-26, 2009.

[20] M. Khalil, C. Enzinger, C. Langkammer et al., "Quantitative assessment of brain iron by $\mathrm{R}(2)^{*}$ relaxometry in patients with clinically isolated syndrome and relapsing-remitting multiple sclerosis," Multiple Sclerosis, vol. 15, no. 9, pp. 1048-1054, 2009.

[21] R. J. Ogg, J. W. Langston, E. M. Haacke, R. G. Steen, and J. S. Taylor, "The correlation between phase shifts in gradient-echo MR images and regional brain iron concentration," Magnetic Resonance Imaging, vol. 17, no. 8, pp. 1141-1148, 1999.

[22] K. E. Hammond, M. Metcalf, L. Carvajal et al., "Quantitative in vivo magnetic resonance imaging of multiple sclerosis at 7 Tesla with sensitivity to iron," Annals of Neurology, vol. 64, no. 6, pp. 707-713, 2008.

[23] Y. Ge, J. H. Jensen, H. Lu et al., "Quantitative assessment of iron accumulation in the deep gray matter of multiple sclerosis by magnetic field correlation imaging," American Journal of Neuroradiology, vol. 28, no. 9, pp. 1639-1644, 2007.

[24] S. A. Smith, J. W. M. Bulte, and P. C. M. Van Zijl, "Direct saturation MRI: theory and application to imaging brain iron," Magnetic Resonance in Medicine, vol. 62, no. 2, pp. 384393, 2009.

[25] E. M. Haacke, Y. Xu, Y. C. N. Cheng, and J. R. Reichenbach, "Susceptibility weighted imaging (SWI)," Magnetic Resonance in Medicine, vol. 52, no. 3, pp. 612-618, 2004.

[26] J. R. Reichenbach, M. Barth, E. M. Haacke, M. Klarhöfer, W. A. Kaiser, and E. Moser, "High-resolution MR venography at 3.0 Tesla," Journal of Computer Assisted Tomography, vol. 24, no. 6, pp. 949-957, 2000.

[27] A. Deistung, A. Rauscher, J. Sedlacik, J. Stadler, S. Witoszynskyj, and J. R. Reichenbach, "Susceptibility weighted imaging at ultra high magnetic field strengths: theoretical considerations and experimental results," Magnetic Resonance in Medicine, vol. 60, no. 5, pp. 1155-1168, 2008.

[28] L. De Rochefort, R. Brown, M. R. Prince, and YI. Wang, "Quantitative MR susceptibility mapping using piece-wise constant regularized inversion of the magnetic field," Magnetic Resonance in Medicine, vol. 60, no. 4, pp. 1003-1009, 2008.

[29] F. Schweser, A. Deistung, B. W. Lehr, and J. R. Reichenbach, "Quantitative imaging of intrinsic magnetic tissue properties using MRI signal phase: an approach to in vivo brain iron metabolism?” NeuroImage, vol. 54, no. 4, pp. 2789-2807, 2011.

[30] J. F. Schenck, “The role of magnetic susceptibility in magnetic resonance imaging: MRI magnetic compatibility of the first and second kinds," Medical Physics, vol. 23, no. 6, pp. 815-850, 1996.

[31] D. Aquino, A. Bizzi, M. Grisoli et al., "Age-related iron deposition in the basal ganglia: quantitative analysis in healthy subjects," Radiology, vol. 252, no. 1, pp. 165-172, 2009.

[32] K. Hopp, B. F. GH. Popescu, R. P. E. McCrea et al., "Brain iron detected by SWI high pass filtered phase calibrated with synchrotron X-ray fluorescence," Journal of Magnetic Resonance Imaging, vol. 31, no. 6, pp. 1346-1354, 2010.

[33] C. Langkammer, N. Krebs, W. Goessler et al., "Quantitative MR imaging of brain iron: a postmortem validation study," Radiology, vol. 257, no. 2, pp. 455-462, 2010.

[34] R. Bakshi, J. Dmochowski, Z. A. Shaikh, and L. Jacobs, "Gray matter T2 hypointensity is related to plaques and atrophy in the brains of multiple sclerosis patients," Journal of the Neurological Sciences, vol. 185, no. 1, pp. 19-26, 2001.
[35] V. Janardhan, S. Suri, and R. Bakshi, "Multiple sclerosis: hyperintense lesions in the brain on nonenhanced T1 weighted MR images evidenced as areas of T1 shortening," Radiology, vol. 244, no. 3, pp. 823-831, 2007.

[36] E. M. Haacke, M. Makki, Y. Ge et al., "Characterizing iron deposition in multiple sclerosis lesions using susceptibility weighted imaging," Journal of Magnetic Resonance Imaging, vol. 29, no. 3, pp. 537-544, 2009.

[37] M. Fukunaga, T. Q. Li, P. Van Gelderen et al., "Layer-specific variation of iron content in cerebral cortex as a source of MRI contrast," Proceedings of the National Academy of Sciences of the United States of America, vol. 107, no. 8, pp. 3834-3839, 2010.

[38] P. Zamboni, R. Galeotti, E. Menegatti et al., "Chronic cerebrospinal venous insufficiency in patients with multiple sclerosis," Journal of Neurology, Neurosurgery and Psychiatry, vol. 80, no. 4, pp. 392-399, 2009.

[39] E. M. Haacke, J. Garbern, Y. Miao, C. Habib, and M. Liu, "Iron stores and cerebral veins in MS studied by susceptibility weighted imaging," International Angiology, vol. 29, no. 2, pp. 149-157, 2010.

[40] R. Zivadinov, C. Schirda, M. G. Dwyer et al., "Chronic cerebrospinal venous insufficiency and iron deposition on susceptibility-weighted imaging in patients with multiple sclerosis: a pilot case-control study," International Angiology, vol. 29, no. 2, pp. 158-175, 2010.

[41] P. Zamboni, "The big idea: iron-dependent inflammation in venous disease and proposed parallels in multiple sclerosis," Journal of the Royal Society of Medicine, vol. 99, no. 11, pp. 589593, 2006.

[42] A. V. Singh and P. Zamboni, "Anomalous venous blood flow and iron deposition in multiple sclerosis," Journal of Cerebral Blood Flow and Metabolism, vol. 29, no. 12, pp. 1867-1878, 2009.

[43] O. Khan, M. Filippi, M. S. Freedman et al., "Chronic cerebrospinal venous insufficiency and multiple sclerosis," Annals of Neurology, vol. 67, no. 3, pp. 286-290, 2010.

[44] M. P. Wattjes, B. W. van Oosten, W. L. de Graaf et al., "No association of abnormal cranial venous drainage with multiple sclerosis: a magnetic resonance venography and flowquantification study," Journal of Neurology, Neurosurgery and Psychiatry, 2010. Epub ahead of print.

[45] F. Doepp, F. Paul, J. M. Valdueza, K. Schmierer, and S. J. Schreiber, "No cerebrocervical venous congestion in patients with multiple sclerosis," Annals of Neurology, vol. 68, no. 2, pp. 173-183, 2010.

[46] V. Worthington, J. Killestein, M. J. Eikelenboom et al., "Normal CSF ferritin levels in MS suggest against etiologic role of chronic venous insufficiency," Neurology, vol. 75, no. 18, pp. 1617-1622, 2010.

[47] P. Sundström, A. Wåhlin, K. Ambarki, R. Birgander, A. Eklund, and J. Malm, "Venous and cerebrospinal fluid flow in multiple sclerosis: a case-control study," Annals of Neurology, vol. 68, no. 2, pp. 255-259, 2010.

[48] A. P. D. Henderson, M. H. Barnett, J. D. E. Parratt, and J. W. Prineas, "Multiple sclerosis: distribution of inflammatory cells in newly forming lesions," Annals of Neurology, vol. 66, no. 6, pp. 739-753, 2009.

[49] B. D. Trapp, J. Peterson, R. M. Ransohoff, R. Rudick, S. Mörk, and L. Bö, "Axonal transection in the lesions of multiple sclerosis," New England Journal of Medicine, vol. 338, no. 5, pp. 278-285, 1998.

[50] E. C. Tallantyre, L. Bø, O. Al-Rawashdeh et al., "Greater loss of axons in primary progressive multiple sclerosis plaques 
compared to secondary progressive disease," Brain, vol. 132, no. 5, pp. 1190-1199, 2009.

[51] J. M. Frischer, S. Bramow, A. Dal-Bianco et al., "The relation between inflammation and neurodegeneration in multiple sclerosis brains," Brain, vol. 132, no. 5, pp. 1175-1189, 2009.

[52] L. Bø, C. A. Vedeler, H. Nyland, B. D. Trapp, and S. J. Mørk, "Intracortical multiple sclerosis lesions are not associated with increased lymphocyte infiltration," Multiple Sclerosis, vol. 9, no. 4, pp. 323-331, 2003.

[53] T. Moos, "Brain iron homeostasis," Danish Medical Bulletin, vol. 49, pp. 279-301, 2002.

[54] J. R. Connor, S. L. Menzies, S. M. ST. Martin, and E. J. Mufson, "Cellular distribution of transferrin, ferritin, and iron in normal and aged human brains," Journal of Neuroscience Research, vol. 27, no. 4, pp. 595-611, 1990.

[55] W. Craelius, M. W. Migdal, C. P. Luessenhop, A. Sugar, and I. Mihalakis, "Iron deposits surrounding multiple sclerosis plaques," Archives of Pathology and Laboratory Medicine, vol. 106, no. 8, pp. 397-399, 1982.

[56] S. M. Levine, "Iron deposits in multiple sclerosis and Alzheimer's disease brains," Brain Research, vol. 760, no. 1-2, pp. 298-303, 1997.

[57] A. Petzold, M. J. Eikelenboom, D. Gveric et al., "Markers for different glial cell responses in multiple sclerosis: clinical and pathological correlations," Brain, vol. 125, no. 7, pp. 14621473, 2002.

[58] K. Mehindate, D. J. Sahlas, D. Frankel et al., "Proinflammatory cytokines promote glial heme oxygenase-1 expression and mitochondrial iron deposition: implications for multiple sclerosis," Journal of Neurochemistry, vol. 77, no. 5, pp. 13861395, 2001.

[59] T. Moos, T. R. Nielsen, T. Skjørringe, and E. H. Morgan, "Iron trafficking inside the brain," Journal of Neurochemistry, vol. 103, no. 5, pp. 1730-1740, 2007.

[60] YA. Ke and Z. M. Qian, "Brain iron metabolism: neurobiology and neurochemistry," Progress in Neurobiology, vol. 83, no. 3, pp. 149-173, 2007.

[61] S. A. Schneider, J. Hardy, and K. P. Bhatia, "Iron accumulation in syndromes of neurodegeneration with brain iron accumulation 1 and 2: causative or consequential?" Journal of Neurology, Neurosurgery and Psychiatry, vol. 80, no. 6, pp. 589-590, 2009.

[62] B. Halliwell, "Oxidative stress and neurodegeneration: where are we now?" Journal of Neurochemistry, vol. 97, no. 6, pp. 1634-1658, 2006.

[63] J. M. C. Gutteridge, "Iron and oxygen radicals in brain," Annals of Neurology, vol. 32, pp. S16-S21, 1992.

[64] G. A. Salvador, "Iron in neuronal function and dysfunction," BioFactors, vol. 36, no. 2, pp. 103-110, 2010.

[65] S. M. LeVine, S. G. Lynch, C. N. Ou, M. J. Wulser, E. Tam, and N. Boo, "Ferritin, transferrin and iron concentrations in the cerebrospinal fluid of multiple sclerosis patients," Brain Research, vol. 821, no. 2, pp. 511-515, 1999.

[66] E. T. Lim, D. Grant, M. Pashenkov et al., "Cerebrospinal fluid levels of brain specific proteins in optic neuritis," Multiple Sclerosis, vol. 10, no. 3, pp. 261-265, 2004.

[67] K. Rejdak, A. Petzold, Z. Stelmasiak, and G. Giovannoni, "Cerebrospinal fluid brain specific proteins in relation to nitric oxide metabolites during relapse of multiple sclerosis," Multiple Sclerosis, vol. 14, no. 1, pp. 59-66, 2008.

[68] C. Sfagos, A. C. Makis, A. Chaidos et al., "Serum ferritin, transferrin and soluble transferrin receptor levels in multiple sclerosis patients," Multiple Sclerosis, vol. 11, no. 3, pp. 272$275,2005$.
[69] N. Abo-Krysha and L. Rashed, "The role of iron dysregulation in the pathogenesis of multiple sclerosis: an Egyptian study," Multiple Sclerosis, vol. 14, no. 5, pp. 602-608, 2008.

[70] R. Van Toorn, J. F. Schoeman, R. Solomons, M. A. Rensburg, and S. J. Van Rensburg, "Iron status in children with recurrent episodes of tumefactive cerebral demyelination," Journal of Child Neurology, vol. 25, no. 11, pp. 1401-1407, 2010.

[71] D. R. Richardson, "Novel chelators for central nervous system disorders that involve alterations in the metabolism of iron and other metal ions," Annals of the New York Academy of Sciences, vol. 1012, pp. 326-341, 2004.

[72] S. M. LeVine and A. Chakrabarty, "The role of iron in the pathogenesis of experimental allergic encephalomyelitis and multiple sclerosis," Annals of the New York Academy of Sciences, vol. 1012, pp. 252-266, 2004.

[73] M. Whitnall and D. R. Richardson, "Iron: a new target for pharmacological intervention in neurodegenerative diseases," Seminars in Pediatric Neurology, vol. 13, no. 3, pp. 186-197, 2006.

[74] T. V. Pedchenko and S. M. Levine, "Desferrioxamine suppresses experimental allergic encephalomyelitis induced by MBP in SJL mice," Journal of Neuroimmunology, vol. 84, no. 2, pp. 188-197, 1998.

[75] K. M. Mitchell, A. L. Dotson, K. M. Cool, A. Chakrabarty, S. H. Benedict, and S. M. LeVine, "Deferiprone, an orally deliverable iron chelator, ameliorates experimental autoimmune encephalomyelitis," Multiple Sclerosis, vol. 13, no. 9, pp. 11181126, 2007.

[76] S. G. Lynch, T. Fonseca, and S. M. Levine, "A multiple course trial of desferrioxamine in chronic progressive multiple sclerosis," Cellular and Molecular Biology, vol. 46, no. 4, pp. 865-869, 2000. 


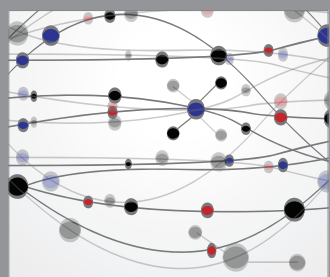

The Scientific World Journal
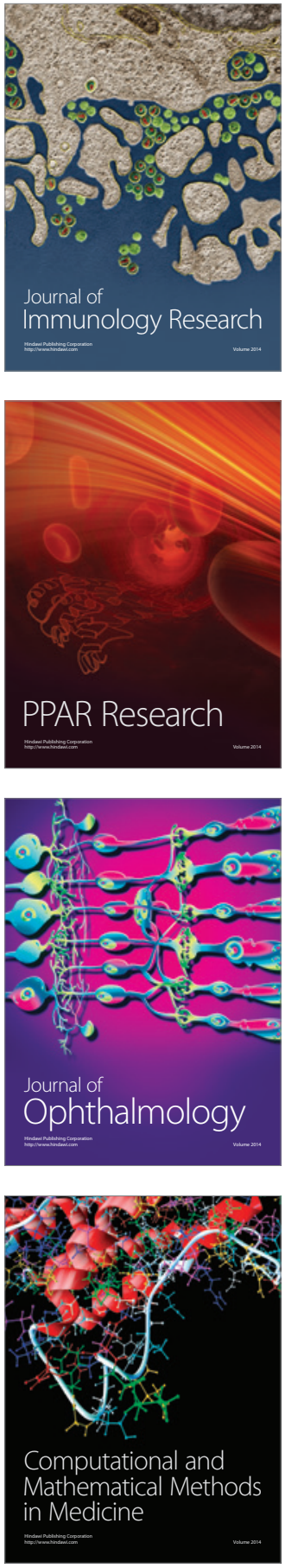

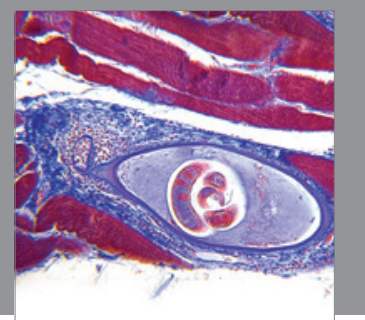

Gastroenterology

Research and Practice
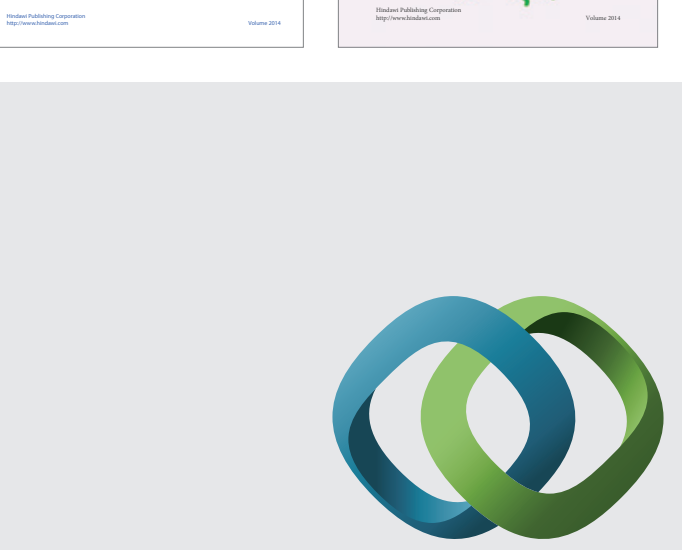

\section{Hindawi}

Submit your manuscripts at

http://www.hindawi.com
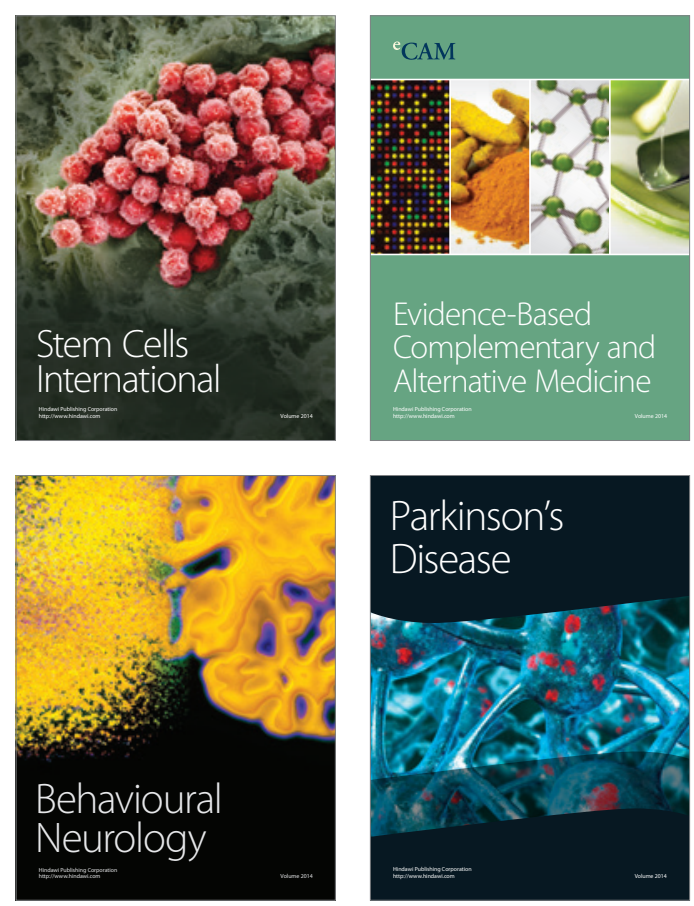

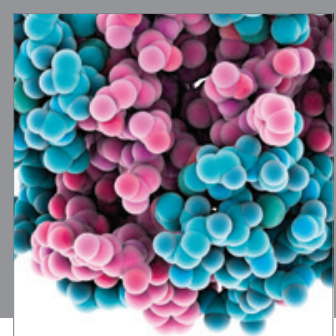

Journal of
Diabetes Research

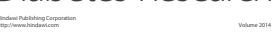

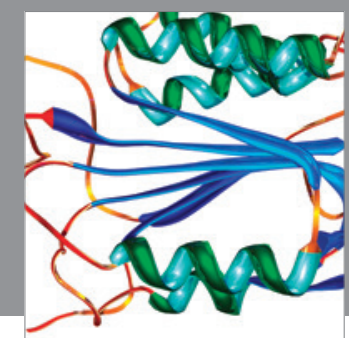

Disease Markers
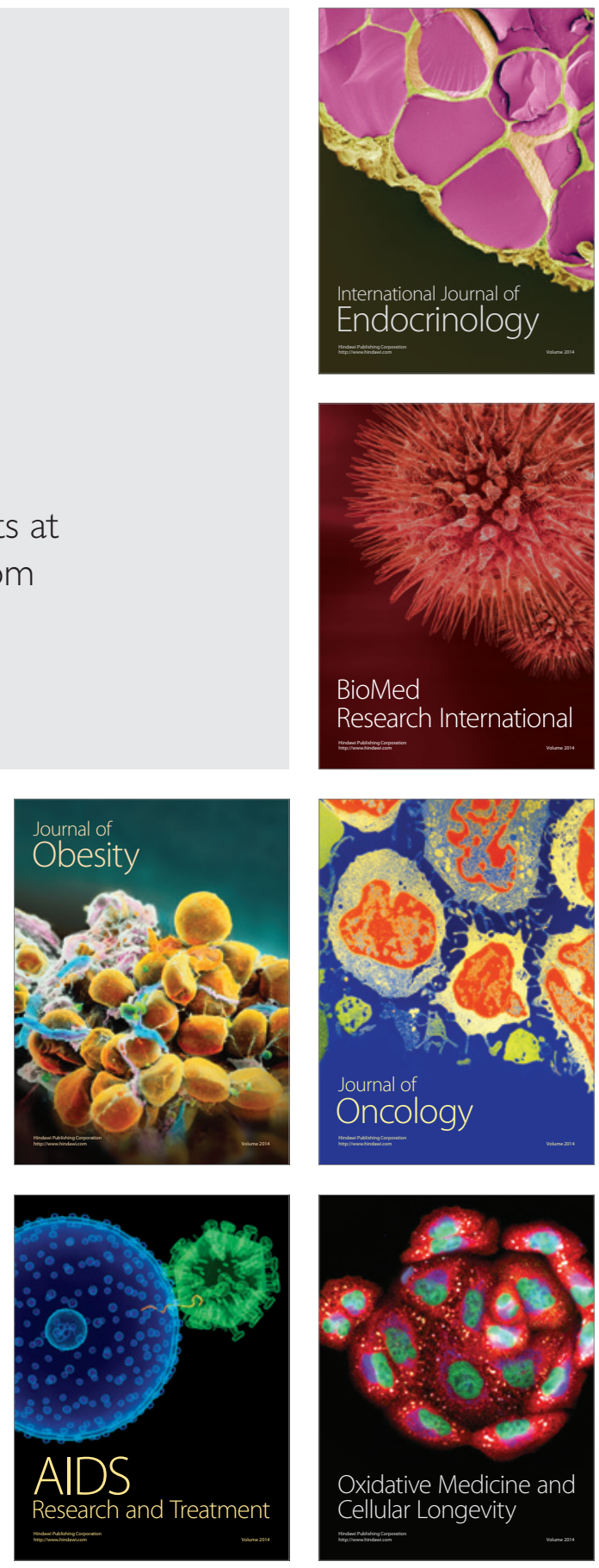